\title{
EL TEATRO ESPAÑOL DE LA SEGUNDA MITAD DEL SIGLO XX EN LOS ESCENARIOS ITALIANOS (1972-1997).
}

\section{Coral GARCÍA RODRÍGUEZ}

\author{
Università di Firenze \\ Grupo Centro de investigación SELITEN@T UNED \\ coral.gr@virgilio.it
}

Resumen: La recepción italiana de las puestas en escena de obras españolas de la segunda mitad del siglo XX se centra en grupos y autores como La Cuadra, La Fura dels Baus, Els Joglars, Els Comediants, José Sanchis Sinisterra, Javier Tomeo, Alfonso Vallejo, Sergi Belbel y Rodrigo García, entre otros.

Abstract: In Italy, the reception of Spanish theater of the second half of 20th Century has focused of primarily on the plays of La Cuadra, La Fura dels Baus, Els Joglars, Els Comediants, José Sanchis Sinisterra, Javier Tomeo, Alfonso Vallejo, Sergi Belbel and Rodrigo García.

Palabras clave: Siglo XX, Teatro español, Representaciones.

Key words: 20th Century, Spanish Theater, Performances. 
Tras haber dedicado mi atención a las representaciones del teatro español de la primera mitad del siglo XX en mi Tesis de Doctorado Europeo (dirigida por el prof. Dr. José Romera Castillo y defendida en la UNED de Madrid en 2000), a partir de la cual he podido preparar y publicar trabajos sucesivos (García Rodríguez, 2002, 2003), ahora ha llegado el momento de ofrecer un panorama no exhaustivo, pero al menos aproximado, de la presencia de nuestro teatro de la segunda mitad de dicha centuria, señalando de inmediato que ha tenido un eco desigual en los escenarios italianos. La intención de este artículo es precisamente recuperar una parte de dicha producción, en la medida de lo posible y a partir de los datos recogidos en revistas y periódicos, citando sólo las puestas en escena de las que he podido contar con reseñas (para más información sobre otras representaciones, remito a la tesis doctoral mencionada).

Pero demos paso a la enumeración de las representaciones en el período que nos ocupa, en la que se dará amplio espacio a los comentarios de los recensores.

1. En abril de 1972, en el Festival palermitano Incontroazione, se puso en escena Metástasis, de Carlos Boves, por el Grupo de Teatro Bubulú, de Madrid. Los sucintos comentarios relativos a esta pieza no son muy halagüeños, y aluden irremediablemente a la situación del teatro en España:

uno spettacolo coraggioso sul piano politico, ma ingenuo su quello teatrale (d'altro canto è ben vero che si tratta di una pièce che la formazione teatrale madrilena neanche si sogna di presentare in patria) (Sipario, junio, 1972: 55).

2. Al mes siguiente, se llevó al escenario Quejío, de Salvador Távora, por la compañía La Cuadra, en el Teatro Eliseo de Roma. En el mes de abril del año sucesivo, el grupo andaluz llegó al escenario del Teatro Fraschini, de Pavía.

Un recensor anónimo explica y reconstruye el espectáculo romano, interpretándolo en una línea de reivindicación político-social del pueblo andaluz:

L'andamento della rappresentazione è assai semplice, poiché l'intento che si sono prefissi i suoi creatori è quello di spiegare, attraverso le immagini e i significati esistenziali che percorrono la storia del popolo andaluso, il valore espressivo e la carica eversiva che anima dall'interno il canto e la danza andalusi, spesso mal conosciuti, soprattutto all'estero, e divenuti specchietto di un esteriore concetto dell'anima spagnola. Tema di fondo dei canti e 
delle danze èl 'oppressione nella quale da tempi immemorabili -e ancor oggi, aggiunge una voce nel commento che precede la rappresentazione- si dibatte il popolo spagnolo. Dieci riti si susseguono dal momento in cui un giovane avanza fino al palcoscenico dal fondo della sala, vestito miseramente, con una lucerna in mano: a lui si aggiungono quattro compagni, miseri come lui, e una donna incinta, testimoni e protagonisti delle cerimonie che vengono ricreate dall'antichità dei tempi dell'Andalusia. Come elemento ricorrente c'è la disperazione del povero e dell'oppresso, la sua mancanza di possibilità a comunicare, a vivere comunitariamente, per un riscatto, con gli altri: lotte, liti, tentativi singoli di vincere la schiavitù della propria situazione si concatenano nella storia dell'uomo, dove i simboli e la realisticità delle situazioni presentate si fondono con effetti talvolta assai belli, talvolta forzati dalla volontà del dire, a tutti i costi, le ragioni intrinseche di una espressione. Alla fine, dopo il canto e i ritmi incalzanti delle danze, solo sottolineati dal cupo rumore dei tacchi che battono sul pavimento, tutti uniscono i loro sforzi in un'azione comune: le corde che tenevano imprigionati i polsi dei giovani e ne condizionavano i movimenti ad un pesante barile, simbolo di dominio, sotto lo sforzo comune si tendono e trascinano il peso prima irremovibile. La vittoria dell'uomo che trova nella lotta comune le ragioni del suo riscatto si compie, e rimane nell'aria come un augurio per una effettiva futura riuscita (Avanti!, 20-5-1972).

En ocasión de la representación de Pavía, por su parte, se advierte al lector del periódico provincial de que este espectáculo da por supuesto un conocimiento cultural del folclore y del denominado "problema andaluz" del que realmente carece el público, aludiendo, además, a García Lorca:

non è da classificare come l'offerta di una serata di tutto riposo, sotto il segno di un divertimento superficiale ed epidermico; si tratta, invece, di una ricreazione, di un clima, di una mentalità, di una realtà, di un costume particolare. Bisogna penetrare la sostanza di un copione immaginato, è chiaro, ma ancorato al vero, e nei dieci quadri che compongono lo spettacolo è necessario vedere, anzi riconoscere, la sostanza di un lamento, la verità di un'ansia e di un anelito ad una vita migliore, la poesia che ha le sue radici nell'anima popolare. Ed a questo proposito ricordiamo il "cante" del rito secondo, Il Martinete, uno dei canti più antichi dei gitani andalusi [...]. Vi si sente il richiamo ad un illustre poeta, a García Lorca, che per questi canti ebbe un grande interesse, e si nota la vibrazione interiore, la necessità di esprimere, con il verso, il sogno e il desiderio, il dolore, l'elevazione alle azzurre soglie della fantasia, dove tutto si placa, ma non si dimentica: si sublima. Ed attraversi il canto, la danza, la musica, Quejío dipana la sua trama, fondendo tragico ed invettiva e grido, e diviene messaggio duro, spietato, frustrazione e liberazione (Provincia Pavese, 17-4-1973). 
Se trató, según la crónaca, de un espectáculo de sugestivo que, sin embargo, no fue apreciado por el tipo de espectador habitual del teatro en el que se puso en escena:

è, ovviamente, nel suo contesto, una rappresentazione non facile, in quanto offre spunti di meditazione e non accontenta i gusti facili. E forse non tutti hanno esattamente afferrate le componenti di questo spettacolo che è stato presentato con dignità, con serietà, con viva partecipazione da parte di tutto il complesso. Riteniamo, però, che il vasto palcoscenico del Fraschini, e lo stesso teatro, siano poco adatti a rappresentazioni del genere, di tipo sperimentale, per un pubblico particolare, per una atmosfera non spettacolare, ma basata sul reciproco impegno fra interpreti e spettatori (Provincia Pavese, 17-4-1973).

En el mes de junio de 1991, se llevó a las tablas del Teatro Argentina de Roma, la versión teatral de Távora de la novela de Gabriel García Márquez, Crónica de una muerte anunciada. A finales de ese mismo año, la obra llegó a la Toscana, en concreto al Teatro Fabbricone de Prato.

La representación romana corrió el riesgo de no hacerse realidad, ya que el sindicato de los trabajadores del teatro había convocado una huelga que, finalmente, se revocó. Pero antes de que se alzase el telón, un sindicalista leyó un comunicado a los espectadores y periodistas presentes en la sala, pidiendo a los políticos que salvaran el teatro, ya que, por una serie de circunstancias precisamente de índole política, se encontraba sin director artístico, y podía perder una subvención de más de tres mil millones de liras y, por consiguiente, estaba irremediablemente destinado a una "muerte anunciada".

Me parece pertinente recordar aquí que el punto de partida del trabajo de Salvador Távora y de La Cuadra surgió del convencimiento de que "nuestra actualidad estaba fuertemente falseada y ocultada por sólidos tópicos":

una corriente traumatizadora había hecho de todo lo andaluz, y de los andaluces, de los que cantábamos y de los que no cantaban, instrumento utilizable para poner una careta alegre y colorista a un pueblo triste y sin color. [...] Había que poner todo "esto" en su sitio, y teníamos que hacerlo nosotros [...]. Había que "mostrar" la verdad del retorcido proceso a todos los traumatizados, andaluces y no andaluces, a los que son -éramos, ignorándolo- parte proyectora de la careta (Villanueva, 1992: 502-503). 
Pero ese pueblo triste y dramático presente en los espectáculos de Távora ya lo conocía el público a través de las obras de Lorca, relacionándolo con retraso y mentalidad arcaica (con la defensa del honor, tan conocido a través del teatro del Siglo de Oro), con la España de la dictadura, con la España negra. En definitiva, esa "nueva" visión del pueblo andaluz no era tan nueva, al menos no en el extranjero, con lo cual La Cuadra no ha hecho otra cosa, en mi opinión, que contribuir a la expansión del tópico que teóricamente pretendía atajar.

Y así se pone de manifiesto en las palabras de un cronista, donde esta visión de Andalucía se identifica, no sólo con Latinoamérica, sino también con el "profundo sur" italiano:

Questa tragedia [...] si fonda sulla incultura della vendetta e dell'onore, che avvicina i latinoamericani di Márquez non solo all'Andalusia, alla Spagna araba di Salvador Távora, ma anche al nostro più profondo sud, alle nostre storie d'onore e di tradimenti, a quegli omicidi una volta giustificati non soltanto dal tribunale popolare, quello della gente, ma spesso anche da quello legale, che, involontariamente, spingeva e giustificava la vendetta (Il Giornale d'Italia, 13-6-1991: 28).

Maurizio Giammusso se detiene a comentar una característica común a todos los espectáculos de Távora, desde Quejío hasta Crónica de una muerte anunciada, cuya ambientación no puede ser más andaluza:

In ogni suo spettacolo [...] racconta la storia e i colori della sua gente andalusa, fin da quando attraversò l'Europa con Quejío [...]. Ora [...] va cercando una luce andalusa anche fra le pagine di Márquez. [...] I fratelli Vicario [...] vanno cercando Santiago Nasa [...]. L'assassinio è annunciato, [...] atteso dalla piccola gente che si chiude dietro le finestre nel caldo sole della controra; non scuote più di tanto l'indifferenza della baffuta polizia di paese, non turba la festa radiosa del clero locale che si prepara ad accogliere il vescovo. Un senso tragico dell'onore, una mistica religiosa che ha più cura della morte che della vita, un fatalismo arreso alla forza ineluttabile della violenza sono l'anima del racconto e allo stesso tempo le forze ineluttabili che spingono senza neppure troppa fretta le mani omicide. [...] In una tragedia della quale si sa tutto fin dall'inizio quel che conta è ovviamente lo stile: García Márquez grazie alla sua scrittura umbratile e semplicissima ha conquistato il Nobel. Távora ha cercato di creare un'analogia stilistica, fatta di poche frasi gridate e ripetute, di un ambiente sonoro di grande suggestione (Il Corriere della Sera, 15-6-1991). 
Y Giorgio Prosperi advierte de la necesidad de liberarse de la fascinación de la novela del Nobel colombiano para poder entender la operación realizada por el director: "Távora ha tradotto l'evento letterario in evento scenico". Este crítico, además, plantea una cuestión que me parece interesante para relacionar a Márquez, Távora y Lorca, en lo que se refiere al sentido trágico que mueve el personal mundo artístico de los tres artistas:

la singolarità del racconto di Márquez consiste nell'anticipare, come già avvenuti, gli episodi che poi si realizzeranno nel corso della vicenda. Così il presente [...] contiene già il futuro, che poi puntualmente si realizza, come nella tragedia greca il fato precede lo svolgersi nella vicenda, dando luogo a una lotta ininterrotta tra destino e libertà. Ciò che distingue profondamente la preveggenza di Márquez dal fato greco è l'assenza di una qualsiasi voce premonitrice. Qui il fatale svolgersi degli eventi sembra determinato dalla volontà inconsciamente collettiva di un intero paese, come un cerimoniale di morte, che nelle anime è già accaduto (Il Tempo, 13-6-1991: IX).

Franco Quadri no se muestra satisfecho de los resultados de la trasposición de la obra narrativa al universo teatral o cinematográfico:

il romanzo breve di García Márquez risulta difficilmente trasferibile in altri "media" per il suo stravolgimento della successione temporale, data dall'immanenza del delitto raccontato, presente fin dall'inizio nella memoria di chi racconta, e destinato a continuare e verificarsi prima della sua consumazione effettiva. Il procedimento [...] ha sortito in cinema una macchinosa telenovela italiana; e non più soddisfacente si rivela i trattamento di Salvador Távora per quanto potesse sembrare connaturato il tipo di espressività che il regista da trent'anni pratica nel lavoro con La Cuadra [...]: una sorta cioè di teatro totale, dove la musica, il folclore, le coreografie assumono palmare rilievo (La Repubblica, 13-6-1991).

Francesco Tei, en una reseña dedicada al espectáculo de Prato, también plantea la diferencia existente entre el texto narrativo del colombiano y la traducción teatral del grupo sevillano: 
Sarebbe facile dire -anche noi siamo d'accordo- che c'è un abisso, ed un distacco profondo, tra la creazione letteraria di García Márquez e la sua rivisitazione di Salvador Távora: lo spirito ed il senso della narrazione e della costruzione originale sono quasi del tutto diversi da quelli della pièce, al punto che si potrebbe arrivare a dire che al centro di questa pur fascinosa "traduzione" teatrale c'è proprio tutto, o quasi, quello che non è la Cronaca [...]. E cioè il palpito doloroso e fatale del "circolo vizioso" amore-passione-destino avverso-Morte, con in più una adeguata dose di sangue, lamento, colpa, sensualità, amore di madre (fra tenerezza e ieraticità sacrale di una figura di genitrice-santa), il tutto condito di poesia fatta di voli di colomba e di petali di fiore sfogliati e sparsi. Colpa, crediamo, anche della sensibilità diversa che c'è non solo tra lo scrittore e gli attori-cantanti e ballerini de La Cuadra, ma anche, in genere, tra l'anima latinoamericana e quella spagnola andalusa. $O$ forse, più semplicemente, il clima ed i contenuti assai particolari che caratterizzano l'opera letteraria di Márquez [...] non interessavano Távora, suo "traduttore" per la scena (La Gazzetta di Firenze, 28-11-1991: 22).

Paolo Lucchesini también explica en qué consiste la "traducción" teatral del andaluz, que transcribo a continuación:

Távora ha voluto trasferire la fosca (e ridicola) tragedia dalla ipotetica, sonnolenta provincia colombiana di Márquez alla rovente, languida Andalusia. Racconto esemplare per squarci e frammenti, in bilico fra presente e passato, la Cronaca si è prestata a diventare una sceneggiatura (non è un caso che Márquez scriva per il cinema) e, inevitabilmente, un film che ebbe un buon seguito a Venezia: un segno tangibile di una precipua vocazione spettacolare dell'opera di Márquez, forse una delle meno immaginarie, mitiche, poetiche. Ma se il film di Francesco Rossi seguiva ordinatamente la trama, Távora ha restituito al racconto quegli elementi magici, simbolici, assenti nella Cronaca, mutuati dal folclore di qua e di là dell'oceano, una miscela esplosiva a sensazione [...]. La storia, ben nota -il titolo è perfino finito fra i luoghi comuni-, contempla la tragica fine del sognatore, svagato Santiago Nazar, additato come seduttore di Angela Vicario, andata sposa, non più illibata, a Bayardo San Román e immediatamente ripudiata. Paulo e Pedro, fratelli della disonorata, decidono di lavare l'oltraggio col sangue. Unico a non sapere dell'agguato dei Vicario è proprio Santiago che si lascerà uccidere sulla porta di casa (La Notte, 28-11-1991: VI).

Tras este breve resumen del argumento de la obra de Márquez, aparece plenamente justificado el intéres de Távora por esta pieza tan "lorquiana"; no olvidemos la adaptación del mismo director de Bodas de sangre. Así como Lorca partió de un hecho real para escribir su tragedia, Távora parte de una narración latinoamericana que 
le servirá, también a él, para reconstruir una visión de Andalucía que, me temo, no ha hecho otra cosa que añadir más "sangre" a un tópico que, en el extranjero, suele atribuirse a toda España.

A principios de septiembre de 1992, Távora volvió a los escenarios italianos con Picasso andaluz. La muerte del Minotauro, representada con anticipación respecto de la fecha del estreno español (fijado para el 14 del mismo mes, en ocasión de la Expo de Sevilla), en la vigésima quinta edición del Festival delle Nazioni di Città Castello, dedicado a España. Junto a Távora, también fue representante de nuestro país García Lorca, "un'altro andaluso di razza e fama mondiale", con La zapatera prodigiosa.

Nico Garrone nos explica en qué consistió el espectáculo de La Cuadra:

ritagliato da stralci d'interviste e corrispondenze di Picasso con le numerose mogli e amanti, il filtro deformante è dato dalla nostalgia verso la terra d'origine del pittore nato a Málaga ed espatriato giovanissimo a Parigi durante il franchismo. Una nostalgia e una capacità visionaria nutrita d'indignazione politica e di ricordi infantili, "madeleines" intinte in quel crogiolo al crocevia tra cultura cristiana, ebrea, musulmana e gitana, che si ritrova anche nelle sue ricorrenti ossessioni iconografiche. Richiami autobiografici e citazioni pittoriche procedono di pari passo integrando sacro e profano, i ceri processionali dei "pasos" della Settimana Santa e le incudini dei fabbri, si alternano con accensioni erotiche gitane e nenie funebri (La Repubblica, 5-9-1992: 28).

En otro septiembre de cinco años después, nos encontramos con el último espectáculo de Távora presente en nuestra cartelera italiana, Carmen, nada menos que uno de los mitos españoles más conocidos fuera de nuestras fronteras. El origen andaluz de esa historia real motiva la elección del tema, con la intención de recuperar a la auténtica protagonista:

Non la peccatrice opportunista di Merimée. Né quella di Bizet, sempre della stessa pasta. Arriva la sigaraia della Real Fábrica de Tabacos, così come da più di un secolo la raccontano a Siviglia; la gitana andalusa realmente esistita, che s'innamorò del poliziotto, poi del picador Lucas (non del torero Escamillo che è un'invenzione), e morì uccisa dal primo amante fuori dalla plaza de toros (La Repubblica, 30-9-1997: 46). 
Laura Putti enmarca esta obra en el declarado objetivo de Távora que, desde 1972, año de la fundación de su compañía, parece estar obsesionado por defender su tierra de origen:

I suoi spettacoli parlano di solidarietà, di popoli emarginati, di valori culturali oramai perduti, di coscienza storica. Ha messo in scena Euripide e García Lorca, Márquez e Picasso. E ora si misura con Carmen, [...] "secondo la leggenda primitiva raccontata dalle vecchie sigaraie di Triana", come recita il sottotitolo dell'opera (La Repubblica, 30-9-1997: 46).

Para presentar al público a la "verdadera" Carmen ("È una lavoratrice allegra, libera di amare, non di vendere amore. La nostra Carmen non è frivola, il suo canto di libertà è vero come il suo dolore"), es necesario acabar con la visión presentada por el célebre francés. Así, Távora afirma tajantemente que la Carmen de Merimée no pertenece a la realidad, sino que es fruto de su imaginación y su fantasía:

Fu, nel 1830, la contessa di Montijo, madre dell'imperatrice Eugenia, a narrargli la storia della sigaraia. [...] Merimée cambiò a suo piacimento la leggenda del quartiere sivigliano di Triana. Mise altri personaggi, come l'oste Lilas Pastia. Nessuna donna sarebbe mai andata da sola in una taverna. Solo le prostitute entravano in un locale pubblico senza un uomo. Carmen non era una prostituta. Era una donna che voleva essere libera, essendo povera, operaia e gitana. Un'impresa difficile. La Carmen di Merimée [...] non è la sigaraia della leggenda sivigliana. Per non parlare di quella di Bizet, del 1875. Ma proprio a causa loro, purtroppo, inizia la deformazione dell'immagine dell'Andalusia (La Repubblica, 30-9-1997: 46).

La Carmen de Távora, por su parte, acoge un importante dato histórico que se habría perdido, si no hubieran sido precisamente las cigarreras las encargadas de transmitirlo oralmente junto con la leyenda: la entrada en Triana del general Rafael de Riego, diputado y defensor de la Constitución de Cádiz de 1812, que acabará siendo ahorcado en 1823 en Madrid, por haber votado por la suspensión temporal de los poderes monárquicos.

La introducción de la Historia de España en la historia personal de Carmen resulta especialmente sugestiva, al enmarcar el deseo de libertad amorosa de la protagonista en la lucha de los partidarios de la Constitución de Cádiz. Al final, es como es bien sabido, ambas libertades serán dominadas por las fuerzas reaccionarias del país. 
3. En el mes de junio de 1987, La Fura dels Baus presentó Accions y Suz/o/Suz en el festival Milanoltre, con la contribución del Centro Nacional de Nuevas Tendencias Escénicas y del Ayuntamiento de Madrid.

Agnese Grieco califica de "irreverentes" y "desestabilizadores" a los miembros del grupo catalán, capaces de mezclar lirismo y crueldad sanguinaria. Pero, además, los espectáculos presentados en Milán son un ejemplo de su carácter polifacético:

Accions, [...] più violento e futurista (che dire del cerchio energetico che viene a crearsi tra il pubblico al momento della "demolizione dell'automobile"?), ribadiva, come vuole un teorico di tutt'altra temperie quale Gilles Deleuze, che il teatro può fare a meno della mimesi, che un corpo in scena è un corpo e non lo rappresenta. [...] Suz/o/Suz [...] si avviava per i sentieri più tortuosi del rito, strizzando l'occhio, forse alla tendenza "paleolitica" di un certo nostro cinema futuribile, ma con un'infinita poesia. Ed era in Suz/o/Suz che La Fura mostrava appieno la propria capacità evocativa, il proprio essere una macchina perfetta in grado di dare vita al rito-accadimento teatrale. Con il pregio di non oltrepassare troppo le righe "ideologiche", di mantenere uno spazio di libertà e di gioco che permetteva di "annacquare" il rito. Annacquare anche nel vero senso della parola, poiché per gli spettatori più coraggiosi c'erano a disposizione "davvero" getti d'acqua colorata a loro modo estremamente chiarificatori. I giovani catalani della Fura, insomma, hanno del "fegato e del talento", del fegato nel saper provocare e del talento nello sfruttare a pieno la terribile capacità espressiva di un violento alfabeto visivo (Sipario, noviembre-diciembre, 1987: 127).

En 1997, se suceden los espectáculos preparados por La Fura, incluidos los realizados por encargo ${ }^{1}$. Como, por ejemplo, Sebastian, un espectáculo multimedial que, con la combinación de géneros diversos, prosa, danza, música, canto y el uso de técnicas audivisuales, reinterpreta, partiendo de D'Annunzio, la historia del mártir cristiano, acompañado por la música de Debussy. Parece que la elección del grupo catalán para realizar este espectáculo fue el deseo de acercar al público a la música clásica, de obligar a los aficionados a dejar la butaca y a presentarse en el teatro:

\footnotetext{
${ }^{1}$ La realización de espectáculos por encargo continuará en 2000. En efecto, el grupo catalán ha trabajado para el buscador italiano de Internet Virgilio, durante el Futurshow, de Bolonia, que se celebró en el mes de marzo.
} 
Sappiamo che questo genere di spettacolo ha registrato un preoccupante distacco da parte degli spettatori abituali che preferiscono la musica impeccabilmente registrata su $C D$, eseguita dai loro interpreti prediletti e ascoltabile nell'intimità delle pareti domestiche. Questo Martirio, trattandosi di un'opera che non ha precise indicazioni sceniche, si presta facilmente come materiale idoneo per creare immagini teatrali, introducendo un nuovo concetto di spettacolo musicale che utilizzi immagini in movimento di grande impatto -cinema e videoinsieme a figure ed elementi corporei in aggiunta all'orchestra, al coro e ai solisti (Sipario, julio-agosto, 1997: 23).

Pero el texto, además de ofrecer posibilidades de manipulación de lenguajes distintos, posee, según Mario Mattia Giorgetti, otro mérito decisivo, su modernidad:

Infatti, la storia del giovane ufficiale della guardia pretoriana condannato nel terzo secolo d.C. perché difende le sue idee, non è affatto estranea ai tempi odierni. Sebastiano è un martire paragonabile a Luther King, a Gandhi, a tutti i perseguitati dal nazismo e ad altre vittime di altri poteri totalitari. Inoltre, la musica che Debussy compose per il testo di D'Annunzio è perfettamente valida ancor oggi per una messa in scena di un'opera che riflette le inquietudini etiche e morali della nostra epoca. Infine, la morte di San Sebastiano, ormai, fa parte della cultura dei miti (Sipario, julio-agosto, 1997: 23-24).

Simbiosis, por su parte, contó con un mecenas de excepción, la empresa automobilística alemana Damimler-Benz, que quiso lanzar con ellos el último modelo Classe A. Por supuesto, la entrada era gratuita. Rossella Sleiter nos cuenta en qué consiste la actuación de los miembros de La Fura dels Baus:

Devono ingerire uova avendo le mani legate dietro la schiena. Devono ricoprire di creta la faccia di qualcuno che non vuole, o il corpo di una donna che si divincola, un po' per piacere un po' per orrore. Furetti che replicano l'avventura della nascita, attraversando viscidi e trasparenti tunnel bianchi lunghi quanto un megaintestino messo in piano. Furetti che affrontano l'acqua e il vento, gli altri due elementi dimenticati, oltre alla terra e il fuoco, su cui si basa Simbiosis. Tutto nel mega cubo e in altri piccoli cubi o Cápsulas, trasparenti e mobili in modo che il pubblico possa vedere, ma non toccare. Chi conosce lo stile dei Fura [...] non ha bisogno di altri particolari per capire che cosa Napoli si prepara a ricevere; chi, distratto, non avesse seguito la formazione catalana, né nello spettacolo d'apertura delle ultime Olimpiadi di Barcellona né quando, a maggio, si esibiva in un multimediale e tecnologico Martyr de Saint Sebastien [...] di qualche pennellata descrittiva forse ne ha bisogno. Del resto mica vorremmo raccontare la trama di un tour come "A-Motion", che gioca sulla parola 
motion, emozione e movimento, e su una semplice quanto fondamentale lettera dell'alfabeto, A, che sta per la classe di auto? (La Repubblica Supplemento Il Venerdì, 3-10-1997: 122-127).

Otro de los espectáculos del grupo catalán, Manes, se representó en distintas ciudades italianas (Nápoles, Milán, Florencia y Udine, entre otras), aprovechando locales semi-abandonados, como la florentina ex Stazione Leopolda. Me ocuparé de la reconstrucción de la puesta en escena en este último lugar. Hay que destacar que la prensa florentina anunció la llegada de La Fura con varios días de antelación, e incluso la incluyó entre los grandes acontecimientos de la semana. El 19 de septiembre, daba la bienvenida al grupo en los siguientes términos:

La trasgressiva compagnia catalana celebre per i suoi spettacoli con acqua, fuoco, pigmenti, viscere sarà in scena [...] con Manes, spettacolo tra gioco e ritualità il cui punto di forza è, ancora una volta, la potenza delle immagini, la finzione teatrale esasperata. L'intento è quello di ricreare la percezione schizoide del nostro tempo, la dissimulazione, la violenza. In uno spazio scenico sobrio, talmente neutro da sembrare onirico, attori e pubblico si scambiano $i$ ruoli di "osservante" e "osservato", suono e illuminazione sono studiati per il senso di mobilità dell'evento così come la drammaturgia di Manes è spontanea, sembra nascere sul momento e segue una grammatica della simultaneità, non della continuità (La Repubblica, 19-10-1997: VIII).

Como afirma uno de los miembros del grupo, en este espectáculo no existe un argumento:

Ognuno [...] può vederci ciò che crede. Una volta, uno spettatore, al termine di Manes, in cinque secondi, mi disse: "Ho capito tutto. È uno spettacolo in cui l'attore che è situato più in alto riesce a mangiare un pollo. Altre volte, spettatori più colti, mi hanno parlato per più di due ore, soltanto per commentarmi la prima scena (La Repubblica, 23-10-1997: XII).

En esta ausencia de una historia concreta que se mueva dentro de los límites tradicionales de planteamiento, núcleo y desenlace, insiste Fulvio Paloscia:

Manes [...] è azione pura, un gioco sulla violenza, una macabra e iconoclasta commedia dell'arte dove gli attori, nudi, copulano coi polli e si fanno accarezzare dagli spettatori. Un evento a metà tra rito orgiastico e processione stracciona, un baccanale di sangue, carne e umori, di bestialità, provocazione e dissacrazione. [...] lo spettacolo si svolge in mezzo al 
pubblico, senza vie di scampo, in uno spazio in cui gli attori braccano lo spettatore, lo costringono a far parte dell'evento, anche contro la sua volontà. [...] Provocatorio e crudele, violento e sconvolgente, attraversato da una selvaggia carica erotica. Manes è il grido di guerra di un teatro sempre più assuefatto, narcotizzato. Ed è un'allusione continua a una creazione caotica e mostruosa: dai liquidi organici e non che inzuppano il pubblico (molti, aiutati dal maltempo, ne approfittano per aprire ombrelli e indossare impermeabili) fino alle gigantesche uova che, mosse all'interno dagli attori, inseguono gli spettatori in fuga, fra grida di panico. E sono proprio quelle uova, sospese in aria su un'impalcatura di legno, a inghiottire gli attori nel finale, simbolico ritorno al niente, al grado zero di un'umanità perversa $e$ invasata (La Repubblica, 25-10-1997: 43; Il Patalogo, 21: 240).

Del 23 al 25 de octubre de 1997, Manes se puso en escena en la ex Stazione Leopolda de Florencia, a las diez de la noche. Roberto Incerti comenta el desarrollo del espectáculo:

alcune ragazze [...] durante lo spettacolo -almeno così garantisce un aneddoto- cercheranno protezione fra le braccia del proprio partner, oppure in quelle di uno sconosciuto ... Questo perché gli attori della Fura, durante Manes, sono spesso nudi, toccano il seno ad alcune spettatrici e gettano farina sul pubblico (La Repubblica, 23-10-1997: XII).

Además, el recensor transcribe la opinión del director del grupo, Pera Tantina, aclaratoria donde las haya de la concepción de teatro en la que se basa toda la historia escénica de la compañía catalana:

Quello che mi interessa [...] è mettere adrenalina nello spettatore. Incutergli paura, insicurezza. Il pubblico, di solito, quando va a teatro paga un biglietto: in cambio ha una poltrona in cui si sente protetto. Con La Fura gli spettatori non hanno alcuna protezione e dovranno stare in tensione durante tutto il tempo della rappresentazione. Dovranno cercare di non essere sopraffatti dagli attori che si muovono. Alla fine uno mi può dire: Manes è uno schifo. Va bene. L'importante è che nessuno si annoi (La Repubblica, 23-10-1997: XII).

$\mathrm{Y}$, efectivamente, es imposible que los espectadores se aburran, porque tienen otras cosas en las que pensar, por ejemplo, en cómo "salvarse" de las agresiones del grupo: 
Il pubblico [...] si troverà a vagare, senza platee né palchi, in uno spazio scenico ampio che diventa un ring, una guerriglia urbana dove vengono mangiati polli crudi e dove gli attori, muovendosi fra il pubblico, spargono all'improvviso acqua, polvere, terra, carogne di animali (La Repubblica, 23-10-1997: XII).

Para terminar, transcribo mi propia impresión como espectadora de la última función de Manes del sábado 25 de octubre. Durante la espera previa a la entrada, el público, fundamentalmente juvenil y asiudo de los conciertos de rock, esperaba pacientemente, bebiendo y fumando, mientras echaba un vistazo a una mesa sobre la que se habían colocado vídeos, camisetas y libros del grupo. Por fin la música sugirió que el espectáculo iba a empezar. Los espectadores se colocan, según sus deseos o atrevimiento, más o menos cerca de una estrutura alta, donde un hombre está sentado delante de un recipiente que despide fuego. Por supuesto no hay asientos, y durante el tiempo que dura el espectáculo, el público irá moviéndose, voluntaria o forzosamente a causa de los empujones de los demás, de un lado a otro del local, prácticamente a oscuras. Algunos sentirán que les cae encima harina, líquido (¿agua? ¿leche?) o trozos de pollo crudo. $\mathrm{Y}$ en un momento concreto una mujer se acercará lentamente a una joven espectadora, a la que mirará con ternura para después escupirle en la cara el agua contenida en la boca, con la consiguiente y comprensible sorpresa de la interesada y del resto de los espectadores. Es prácticamente imposible seguir todos los "acontecimientos", dada la aglomeración de gente; incluso hay quien acaba pegado contra la pared. Los más prevenidos asistieron al espectáculo con impermeables (el tiempo climático no sugería la utilización de dicha prenda el sábado día 25 , sino las recensiones previas leídas en los periódicos). Los protagonistas, desnudos, suben y bajan por un estructura a modo de árboles; empujan una especie de barriles en forma de huevo por el espacio compartido por el público; hacia el final del espectáculo, se pronuncian algunas palabras en las diferentes lenguas de los componentes del grupo, los cuales terminarán encerrándose en los huevos, ahora suspendidos en el aire.

4. En el verano de 1973, Els Joglars representaron El joc y Mary d'Ous, en el festival de Spoleto, bajo la dirección de Albert Boadella, con escenografía y vestuario de Yago Pericot, e iluminación de Iokin Cueto. Los intérpretes fueron Albert Boadella, Marta Catala, Lluisa Hurtado, Ferran Rane, Gloria Rognoni, Andreu Solsona y Jaume Sorribas. 


\section{Giorgio Polacco los considera los mejores espectáculos del encuentro:}

La prosa migliore, quest'anno a Spoleto, parlava il linguaggio del silenzio. Non occorre l'ausilio della parola, ai mimi spagnoli di Barcellona. Basta un sorriso o un colpo di tosse, basta l'andatura pigra oppure saltabeccante, basta l'improvvisa serietà dipinta sul volto prima allegro di un clown, o al massimo qualche esile suono onomatopeico, e la magia del teatro mimetico si rinnova, più forte di mille parole roboanti, più imperiosa di cento sermoni didascalici, più "utile" di tanti comizi camuffati da azioni sceniche. Il segreto (e troppo dire la "lezione"?) di questi Els Joglars catalani è proprio nella loro "capacità di contagio" che reclamava Artaud e che Renzo Tian ricordava con indovinata pertinenza. Contagiante è tutto lo sketch, in El Joc, sul lavoro operaio, un occhio al Moloch dello Stakanovismo e un occhio alle miniere basche, tra martelli pneumatici, catene di montaggio, sirene ululanti, e la morte in mille maniere, precipitando da impalcature, sfracellandosi nelle fabbriche vocianti, saltando per aria dilaniati. Ma anche altri quadri posseggono l'identica forza di suggestione e di partecipazione: quello della nascita del mondo, con un Dio metà burlone e metà disilluso, che si fa saltar le cervella, per via di tutto il guazzabuglio che ha combinato; o la sequenza finale, con le mille maniere di uccidere che impiega la cosiddetta "civiltà" moderna: ghigliottina e garrotta, plotone d'esecuzione e sedie elettriche. [...] Pensate ai due personaggi (in Mary d'Ous) che si chiamano l'uno "Issimo", generale-dittatore, collerico e scorbutico, l'altro "Vice", destinato alla successione, e comprenderete bene come la sferza satirica non si limiti affatto a una generica, anche se effervescente "vis comica", ma sia al contrario improntata a una dirompente carica politica. Uomini e donne sono quelli di una società poco più che feudale, dove la condizione della femmina è ancora quella della casalinga che in continuità sbatte le uova di pantagrueliche, eterne frittate. Uomini e Poteri sono quelli di una società repressiva dove le strutture del Comando stritolano la libertà individuale. Come nel gran telone bianco, al finale, che avvolge e sommerge tutti, vittime innocenti divorate da una piovra malefica. Sotto questo lenzuolo bianco di morte sono loro, questi bateleurs eredi di circhi lontani e felici, ultimi saltimbanchi in odio ai Potenti (Sipario, agosto-septiembre, 1973: 54).

Además, el mismo crítico sitúa al grupo dentro de una tendencia internacional, donde, sin embargo, mantiene su propia originalidad:

Stilisticamente il gruppo rivela le ascendenze americane del Living e fors'anche dell'Open, ma con una componente mimica spiccatamente mediterranea e un gusto per i colori accesi e l'atmosfera strampalata che ricordano anche i clowns tristi di Jerome Savary e del suo pazzo pazzo pazzo Magic Circus. Ma non si commetta l'errore di scambiare le risate per evasive frivolezze. Al contrario, sono ghigni sinistri sotto l'effettaccio del contrappunto ironico (Sipario, agosto-septiembre, 1973: 54). 
Un espectador de excepción, Angelo Maria Ripellino, también dedicó una reseña al espectáculo $\mathrm{El} \mathrm{joc,} \mathrm{que} \mathrm{describió} \mathrm{en} \mathrm{los} \mathrm{siguientes} \mathrm{términos,} \mathrm{tratando} \mathrm{de} \mathrm{reconstruir}$ una línea argumental:

El joc [...] [è] uno spassoso concerto di biomeccanica, un canzonatorio centone di sei numeri mimici, uno scanzonato teatro da piazza e mercato di caricature. [...] La strategia dei Joglars è tutta imperniata sul Contrattempo. [...] L'automatismo produce disperatissimi errori. Un operaio che manovri una fiamma ossidrica brucerà per sbaglio un compagno. Un boia, nell'alzare la scure, troncherà per sbaglio un estraneo. Lo sforzo umano è incrinato continuamente da lugubri spasmi di risa. Sia che beffeggino la meccanicità del lavoro o raccontino a scorsi, in un retablo di rapidi accenni gestuali, tra spari mischie, marcette, rataplan tambureschi, la storia della condanna a morte -anche i temi luttuosi offrono il destro ai Joglars per fughe di burle e di gags. [...] Ma il culmine dello spettacolo è l'astuta pantomima della creazione dell'uomo, una ridanciana Genesi da cartellone di fiera. Dio, prestigiatore dai lunghi capelli biancastri, fabbrica un ballonzolante Adamo inclini ai flamenchi e una nera bertuccia. Poi, per placare le voglie del protoplasto, modella con una sua costola una monachina in gramaglie dallo spagnolesco sussiego. Ma Adamo deluso la scaraventa nel baratro ed è giocoforza per l'illusionista plasmargli una donna procace, con un cerchio da tirassegno sul ventre e una freccia che indica la cotalina. Dopo aver loro mostrato su due alberi da sillabario le mele del Bene e del Male, Dio stesso indossa una verde maschera di serpente con lingua di Menelik. Accade l'ineluttabile. Infilata una maschera di istitutrice zitella, il Creatore sdegnato li scaccia dall'Eden assieme alla scimmia. Mani sulle pudenda, essi sloggiano. Il primo uomo è costretto a zappare la terra. Poi nuovo colpo di scena. Eva si emancipa, lavora come dattilografa, Adamo trova un impiego di autista. A Elohim sconvolto non resta che tirarsi un colpo (Ripellino, 1989: 216-217).

Para continuar sucesivamente con la descripción de "lo que ocurre" realmente en el escenario, y proporcionándonos datos relativos a la pura puesta en escena:

su una rossa rotonda pedana inclinata sei funamboli in neri calzoni di cuoio (due donne e quattro uomini) imbastiscono trame di giocoleria: a gatto e topo, a cane e gatto, ai sorci che ballano, fitte sequele di inseguimenti e cadute, accompagnandole con borbottio, borborigmi, smozzicate parole di canzonette e fumetti. A volte quella destrezza mimetica genera congegni assurdi, che fanno pensare ai racconti di Julio Cortázar, a volte invece si impiglia nel verosimile. [...] In un numero tutto ghingheri e vezzi, ciangottio, risatine, finti lanci di torte, $i$ catalani funamboli contraffanno i giuochi erotici di due coppie, mimandone passo a passo il decorso dai convenevoli all'avvinghiamento (Ripellino, 1989: 216-217). 
El 21 de mayo de 1979, Els Joglars llevó a las tablas M-7 Catalonia, en Milán, y un mes más tarde llegó a Florencia. Alvaro Vaccarella explica el significado del título, resumiendo también "l argumento" de este espectáculo:

M7 nell'ordinamento mondiale di un futuro non molto prossimo, è la sigla con cui verrà indicata la Catalogna. Di questa regione, sono originari i quattro vecchietti (tre uomini e una donna) che due studiose di antropologia mostrano al pubblico di una società asettica e ossessivamente nitida. Vengono così analizzati gli atteggiamenti quotidiani di una cultura "arcaica" attraverso le cervellotiche e talvolte ridicole argomentazioni delle due studiose dagli atteggiamenti rigidamente simmetrici e controllati e dal sorriso costantemente inespressivo. [...] [I] superstiti della civiltà catalana affrontano, con furbizia contadina e con atavico sospetto, la rappresentazione della cultura (termine inteso come Pasolini lo andava proponendo) della vita di tutti i giorni. Dall'atteggiamento che viene assunto al passaggio di una ragazza, al sesso, dalla preparazione della paella, al rito della messa, senza alcuna soluzione di continuità, trasformando la mensa in un altare. Lo spettacolo si chiude con brusca ambiguità: gli individui diventano immobili: pronti per la vetrina di un museo o segno che qualcosa si è inceppato nella perfetta macchina del futuro? (Sipario, agosto-septiembre, 1979: 46).

5. En la primavera de 1979, la compañía La Claca, dirigida por Joan Baixas, puso en escena Mori el Merma, en el Festival Internazionale di Marionette e Burattini de Parma, y en el festival Milano Aperta.

Alvaro Vaccarella centra su interés en la colaboración de Joan Miró en la realización de los los muñecos utilizados en esta sátira contra el la prevaricación del poder. Pero dejemos que sea el mismo cronista el que nos adentre en el momento histórico en el que tiene lugar la representación, cuando la democracia española está empezando a dar sus primeros pasos, pocos años después de la muerte del dictador:

Mori el Merma è anche una profonda ricerca verticale di una compagnia che muovendo da un'ottica culturale di respiro europeo si rivolge all'interno della cultura catalana per vivificare antichi umori, remote tracce di un popolo che va riallacciando una continuità storica $e$ antropologica congelata negli anni della dittatura. Da qui il titolo, che significa Abasso il Merma, un grido lanciato dai bambini di Barcellona il giorno del Corpus Domini quando passa per le strade la maschera di Merma, il tiranno [...]. E come se improvvisamente una canzone iniziata quarant'anni fa e lasciata in sospeso per tutti questi anni avesse ripreso a suonare, mantenendo intatte freschezza e vitalità. È come se la stagione dei poeti e degli artisti 
che animarono la Spagna repubblicana continuasse e si sviluppasse ogni sera con la rappresentazione di Mori il Merma. [...] la Spagna, [...] in questo nuovo clima di libertà, torna a fare udire la sua voce al mondo. E questa volta non dall'esilio. [...] La vicenda di Merma (ovvero Ubu, ovvero del fantoccio-che-rappresenta-il-potere), si snoda in tappe successive. L'incontro dei pupazzi, il momento di splendore, gli intrallazzi della corte, l'approccio sessuale, l'intervento delle maschere piatte, la morte del Merma, il dolore vero e quello provocato dalla perdita dei privilegi, l'apoteosi finale. Risultato più felice non ci si poteva attendere (Sipario, mayo, 1979: 16).

6. En el mes de abril de 1990, La Tartana llevó al escenario del Teatro dell'Elfo de Milán, la adaptación de Guillermo Heras de Ribera despojada, Medea material, Paisaje con figuras, de Heiner Müller.

Valerio Fantinel, tras mencionar la extraña que le produce la reducida presencia del teatro ibérico en suelo italiano, nos aclara inmediatamente el enfoque de esta pieza alemana a manos de su director, Carlos Marquerie:

Müller viene fatto ruzzolare, letteralmente, fra le gambe degli spettatori dal collettivo di lavoro La Tartana [...], con un rigore registico accigliatissimo, denso di richiami visionari e citazioni novecentesche, non tutte di prima mano. [...] Il regista [...] ha puntato sul tema di fondo del viaggio, su paesaggi del mito, delle strutture profonde, collegate fra loro da un filo d'Arianna, tenuto saldamente in pugno dal gruppo. Si fa densa di riferimenti la metafora dell'imbarco e dello sbarco, delle fatiche dell'emigrazione, delle avventure militari di eserciti conquistatori: $e$ i miti si chiamano Medea, gli Argonauti, la Colchide, Giasone. [...] Storia e mito si sovrappongono (Artaud e Nietzsche), nel concatenato splendore dell'iconologia di riferimento (la scena molto spagnolesca del Trionfo della Morte), alle ibridazioni verbali, all'esemplare frammento centrale che fa ammutolire la Storia e che ci viene urlato, in tutta la sua incandescenza, dal volto teso e profetico della brava Sian Thomas: sprofonda e ci fa sprofondare nel Mito delle Madri, nel sotterraneo alveare dei nostri primordiali istinti (Sipario, mayo-junio, 1990: 131).

El recensor también nos ofrece una descripción de la escenografía, acompañada comentarios relativos a la gesticulación, la voz, el sonido y la música, que nos permite completar la reconstrucción de dicha puesta en escena: 
Lo spettacolo è rappresentato su una pedana centrale chiusa ai lati e di fronte da un emiciclo di sedie strapiombanti. Sul fondo della pedana un rialzo con due scivoli ai lati, delimitati da pali congiunti fra di loro da corde su cui vengono innalzate bianche tele (vele), o panni stesi ad asciugare, dopo essere stati lavati e sbattuti da tre donne-furie che mettono a repentaglio, impietosamente, la stessa incolumità degli spettatori. [...] Lo spazio è costruito tutt'attorno a uno specchio d'acqua, percorso da un miniveliero, trasposizione in macrostrutture del viaggio per mare che si riproduce in macrostrutture attraverso il dondolio, il beccheggiamento mimato dagli attori. Da quel miniveliero si irraggia tutta una serie di gesti, movimenti, canti corali di masse in azione, di rumori quasi impercettibili che si fanno sonori ed evocativi, come lo srotolamento di una carta geografica; la sabbia che viene sparsa sul pavimento e scricchiola e stride sotto gli scarponi degli Argonauti, alternata a tonfi di bastoni, di piedi, di corpi, di scivolamenti in acqua. Tutto un repertorio di segni e segnali che costruiscono l'aura della scatola magica e la proiettano in altre dimensioni, là dove lo spettacolo cresce su se stesso, per sua forza, ibridato da voci e da suoni (spezzoni musicali del Tannhaüser wagneriano) che ne simulano il contenuto: la rabbia, la paura, la strage, ecc. Una rilettura contextúale di Müller, in cui sono stati estrapolati spezzoni al color bianco, in una recitazione che punta sul corpo e sul collettivo come un tutt'uno che produce soluzioni plastiche di grande suggestione visiva, erodendo però spazio alla parola, al testo mülleriano. Si è voluto scarnificare il testo per arricchire lo spazio sonoro, disarticolare i corpi, devastati dalle malattie, dalla storia subita, dalle repressioni, dalle punizioni, dalle torture, dagli sradicamenti. Assumendo il gesto come segno estremo, si è voluto razionalizzare l'orrore del tragico, farlo diventare una costante del nostro orizzonte storico (Sipario, mayo-junio, 1990: 131).

7. El 21 de julio de 1990, se lleva al escenario la célebre ¡Ay, Carmela!, de José Sanchis Sinisterra, pero adaptándola para que pareciese una obra hecha por y para italianos. La obra seguirá representándose durante el año siguiente (en los meses de enero y agosto) en julio de 1994, en noviembre de 1995 y en 1996 (en noviembre y diciembre).

El grupo Pupi e Fresedde explicó en qué consistió la italianización del texto, (que contó con la aprobación del dramaturgo valenciano), y la razón de ser de la adaptación de Savelli:

Nel testo originale la vicenda si svolge in Spagna nel 1938 a Belchite, villaggio simbolico degli effetti della ferocia distruttiva della guerra civile spagnola, le cui rovine vengono ancora oggi visitate come un monumento nazionale. Lo spettacolo poteva essere ambientato indifferentemente in un qualsiasi luogo durante una qualsiasi guerra senza per questo perdere 
nulla della sua accorata denuncia. Ma siccome la memoria e lo sdegno non si nutrono d'astrazioni ma di tanti piccoli gesti concreti, il regista d'accordo con l'autore ha trasferito l'azione nell'Italia del 1944 nei territori occupati dalle armate tedesche, pensando che un'ambientazione più prossima e familiare avrebbe aiutato il pubblico italiano a recepire con più naturalezza la tensione morale che sottende la tenue vicenda di Carmela e Paolino. La stessa considerazione vale sul piano estetico. La parte centrale del testo originale è occupata dal ricordo dello spettacolo di varietà improvvisato da Carmela e Paolino per le truppe d'occupazione. Sinisterra ha qui trasfuso nel testo con grande abilità un insieme di riferimenti teatrali tipici del più tradizionale teatro leggero spagnolo. Tali riferimenti non sono affatto dissimili dai nostri. È impressionante constatare l'esistenza di modelli comuni a tutte le culture popolari pur nel variare dei contesti geografici. Ma nel rispetto di quel particolare tono, o gesto o colore, che fa una determinata cultura unica e irrepetibile, anche qui è stata compiuta un'operazione di adattamento ricostruendo uno spettacolo parallelo a quello previsto nel testo originale, un nuovo spettacolo che dell'altro rispetta le cadenze e il procedere ma i cui riferimenti stilistici diventano senza mezzi termini tutti italiani: Rascel, Nino Taranto, Totò, la Magnani, Macario, Pisani e Cioffi eccetera (Il Patalogo, 14: 105).

Mauro Martinelli también se hizo eco del cambio de contexto, especificando dónde se ambienta la versión italiana:

Nel testo originale la vicenda si svolge in Spagna nel 1938 in piena guerra civile, mentre la trasposizione italiana è ambientata nel 1944 in un oscuro paesino abruzzese occupato dalle armate tedesche, Pratolapeligna (Sipario, septiembre, 1991: 55).

El mismo crítico resume el argumento de la obra, permitiéndonos comprobar que, efectivamente, se trata de la "misma historia" narrada por Sinisterra, donde se han mantenido también los nombres de los protagonistas:

Carmela e Paolino due guitti di varietà che durante la notte, a causa della nebbia, hanno varcato senza saperlo le linee nemiche, vengono catturati dai tedeschi e costretti a recitare il loro spettacolo senza scene e con costumi improvvisati, ultimo diversivo per alcuni soldati polacchi prigionieri che verranno giustiziati dopo lo spettacolo. Mentre Paolino, pavido e impaurito dalla prepotenza delle divise, tenta di dissuaderla, Carmela urla contro i tedeschi tutta la sua rabbia per un numero che è costretta a recitare come ultimo atto di derisione verso i soldati polacchi e viene uccisa sulla scena da un ufficiale nazista. Lo spettacolo comincia nel buio della sala con l'incontro tra Paolino e il fantasma della sua compagna, dopo che questa 
lo ha lasciato solo. Carmela riappare e racconta di un al di là dove le persone lentamente si sfocano fino a scomparire, e tenta di ricordare insieme all'amato i bei momenti che hanno vissuto insieme fino a quella tragica sera. Ma Paolino la sfugge, la maledice per ciò che ha fatto lasciandolo solo, salvo poi ingelosirsi quando lei gli racconta delle attenzioni che, "di là", le rivolge Igor, il romagnolo. Carmela addenta una mela, ma non sente alcun sapore; ormai, di lei, non esiste che il ricordo. Ed è il ricordo del loro ultimo spettacolo che li accompagna insieme, in un flash-back che in un lento crescendo arriva a far rivivere attimo per attimo la loro ultima sera sul palcoscenico (Sipario, septiembre, 1991: 55-56).

No se trata, entonces, de un texto percibido como español por el público florentino (ha desaparecido la ubicación espacial y temporal hispánicas, así como las referencias histórico-culturales), sino como un producto dramático que cuenta un fragmento de la historia italiana del siglo XX.

La compañía florentina ha ido acumulando éxitos durante los ocho años en los que ha tenido esta obra en cartelera tanto en Italia como en el extranjero: desde Bélgica, Francia y Portugal, hasta Argentina y Turquía, sin olvidar la misma España. Por si fuera poco, en la capital turca se realizó una versión a partir de la adaptación italiana. El Teatro de Rifredi, además, recibió el 3 y 4 de noviembre de 2000 a la compañía del Teatro de Guerra de Sarajevo, que puso en escena la obra de Sinisterra en su lengua eslava, bajo la dirección de RobertRaponja. Pocos días después se llevó al escenario en el Teatro di Sardegna de Cagliari.

8. A finales de septiembre de 1990, el grupo Els Comediants participó en el festival ExtraMura '90 de Florencia con La nit, espectáculo que Mauro Martinelli interpreta bajo el espejismo de la "movida", como algo a medias entre Almodóvar y Buñuel:

Gnomi, folletti, lune di cartone, cavalieri senza macchia e diavoli senza paura, si agitano in una "movida" fatta di ombre e di odori, materializzata sotto un cielo stellato che si erge sopra le teste degli spettatori e li copre con il suo caldo abbraccio lungo tutta la rappresentazione. È questo un gioiello surreale, e la netta sensazione che l'amore per il teatro non sarà mai mal risposto (Sipario, noviembre, 1990: 86).

9. El 1 de marzo de 1991, se puso en escena Amado monstruo, de Javier Tomeo, bajo la dirección de Angelo Savelli. Franco Quadri explica cuál es el tema de esta 
adaptación teatral de la novela homónima, asignando curiosamente a los españoles una actitud masculina como "el mamismo" que el imaginario extranjero suele asociar más con los italianos:

Spagnolissimo è il tema, ovvero l'ideologia ossessiva della mamma, simbolo carnale di ogni repressione e quindi anche in astratto del potere. Non abbiamo a che fare con lei fisicamente, ma con la sua ingombrante proiezione, odiata fino al desiderio di sopprimerla da uno dei due protagonisti maschili, che ne è vittima, e idealizzata dall'altro che l'ha perduta da ragazzo. La sua immagine, che condiziona dialoghi e comportamenti, potrebbe anche non esistere nella realtà, comunque da lei deformata, come in quelle ipertrofie fantastiche tante volte incontrate nei film di Ferreri o di Berlanga, o nelle deviazioni surrealiste delle commedie di Kopit o di Copi (Il Patalogo, 14: 105; La Repubblica, 19-3-1991).

El director de la obra ofrece una interpretación psicoanalítica, en la que la madre es una creación de los protagonistas de la que se sirven para autojustificarse:

In Amato mostruo forse entrambi i personaggi s'inventano la madre per giustificare la vita impossibile che conducono: quella di Juan, soffocata dall'inattività $e$ solitudine, ma anche quella di Krugger, perduto come un bambino nell'universo degli uffici, della puntualità, della produttività (Il Patalogo, 14: 105; La Repubblica, 1-3-1991).

Ugo Ronfani también insiste en dicha interpretación, coincidiendo ambos, entonces, con las afirmaciones de $\mathrm{Tomeo}^{2}$, acerca del tema de la pieza en cuestión:

La vicenda [...] propone semmai, con traslucido realismo magico, una problematica psicopatica che senza divagazioni psicanalitiche collega le neurosi simmetriche di due celibi rimasti bambini, in un'aura che ricorda Kafka e Buñuel, non senza gli influssi di Pessoa, di Pinter, di Handke (Il Patalogo, 14: 105; Il Giorno, 18-3-1991).

10. El cero transparente, de Alfonso Vallejo, se representó, en el mes de mayo de 1992, en el Teatro della Quattordicesima de Milán. Felice Cappa presenta al autor como uno de los dramaturgos más interesantes de la España contemporánea, cuya

${ }^{2}$ El autor, en una entrevista de A. Agnolotti, afirmaba que "Non vi è motivo edipico nei miei testi ma piuttosto una viva problematica psicopatica" (Il Patalogo, 14: 105). 
destreza más sobresaliente sería la ruptura de las estructuras del tiempo y del espacio (La Notte, 16-5-1992). El cronista, además, nos ofrece un sucinto resumen de la obra que propongo a continuación:

Quattro personaggi salgono su un treno per una meta mitica, sempre più incombente e sempre più simile all'ultimo viaggio. Paradossalmente, proprio quando le loro vite stanno per concludersi,sentono di potersene appropriare. Tutti hanno alle spalle sogni deliranti e vite devastate. I protagonisti che appaiono affetti più da ipersensibilità che da vere e proprie malattie, intessono relazioni violente e tenerissime, comunque sempre sincere. Ad essi si contrappongono i presunti paladini di una razionalità fragilissima, pronta ad entrare in crisi appena viene messa in atto (La Notte, 16-5-1992).

11. De Sergi Belbel se representan dos obras a finales de 1994: Caricias, en dos ocasiones, en el marco del Intercity Festival florentino dedicado a Madrid; y Talem, en el Teatro Anfitrione, de Roma. Luigi Testaferrata, define la primera pieza en los términos siguientes:

dieci episodi di vita quotidiana che Belbel -sulla scia del Girotondo di Schnitzler, ma con pazzia beckettiana- inventò nel 1991 e che solamente ora [...] arrivano in Italia. Dieci scene di vita familiare legata l'una all'altra come vagoni di un treno che sta per saltare in aria; dissacrante delizia da cogliere al volo (La Voce, 2-10-1994).

Cristiano Felice, por su parte, comenta Talem:

L'originalità dello spettacolo si impone attraverso una serie di intelligenti espedienti che, pur centrando in pieno le cause del malessere che portano all'incapacità di comunicare tra gli individui, tuttavia riescono a porre lo spettatore in uno stato di continua attenzione. L'intreccio narrativo, che vede i quattro attori imbrigliati in una situazione che ruota di fronte all'esigenza di "inaugurare" un letto matrimoniale e resa complicata da improvvisi impegni che costringono una delle due coppie all'impossibilità di portare personalmente quest'atto a compimento, costituisce uno degli elementi originali della storia (L’Umanità, 27-11-1994).

El recensor relaciona esta obra con la incomunicación en la sociedad contemporánea, señalando que el autor catalán ha sabido escapar del peligro de llevar al teatro un argumento con el que se corría el riesgo de caer en la banalidad. 
Dos años más tarde, Sergi Belbel volverá al teatro florentino, donde se "leyeron" dos piezas en el mismo día: Morir, "protagoniste sette coppie di personaggi tipici dell'immaginario metropolitano", y Después de la lluvia, "che parla ancora di convivenza, ma in chiave fantascientifica: in una città dove non piove da due anni, le autorità hanno bandito il fumo lasciando esplodere neurosi collettive" (La Repubblica, 12-10-1996).

Ambas obras serán traducidas y publicadas en el número correspondiente de la revista del festival (Belbel, 1996a, 199b).

12. Rodrigo García inauguró el ya mencionado Intercity festival de 1994, del Teatro della Limonaia de Florencia, con una obra, Notas de cocina, donde la comida es interpretada como metáfora sexual. En efecto, Giovanni Raboni señala que nos encontramos ante la célebre y paradójica metáfora de la angustia o de la lucha por la vida y la posesión amorosa que se esconde detrás de la relación con la comida (Il Corriere della Sera, 20-9-1994). Stefania Chinzari explica que el germen de la obra es genuinamente italiano:

un ironico libro di ricette attribuite a Leonardo da Vinci, Note di cucina, recentemente pubblicato in Spagna e salutato da gran successo. Il colpo di fulmine è scoccato subito, magari non imprevedibile, vista l'attrazione fatale tra arte e cucina che percorre la cultura spagnola, dalle celebrate ricette di Vázquez Montalbán ai prosciutti di Bigas Luna. Il resto l'ha imbastito García, mescolando un personale gusto per il sarcasmo e la provocazione; il fatto che già la sua compagnia madrilena si chiami macelleria; un denunciato autobiografismo, nonché i dieci anni passati in pubblicità ("è stato facendo il creativo che ho imparato a odiare i ristoranti: è sempre attorno a un tavolo colmo di cibo che si prendono le decisioni meno innocenti") (L’Unità, 19-9-1994).

Recientemente el autor argentino ha vuelto al mismo teatro con dos piezas: $L a$ historia de Ronald, el payaso de McDonald's y Borges. En ocasión de la puesta en escena, tuvo lugar un encuentro con el autor, entrevistado por Franco Quadri, en el que también se presentó la edición de varias piezas traducidas al italiano (García: 2003).

13. Como no podía ser de otro modo, los cronistas suelen hacer referencia a la situación política en la que se encuentra España al momento de la representación italiana (así, se alude a la imposibilidad de representar determinadas obras durante el 
período franquista, y sucesivamente se celebra la recobrada libertad al llegar la democracia).

Resulta evidente, además, la pujanza y el interés suscitado por los grupos de teatro catalanes (de los que se recuerda su conexión con il Living y el Open, por ejemplo), en detrimento de los dramaturgos con nombre y apellidos propios. Cito como ejemplo paradigmático el caso de José Luis Alonso de Santos, creador de un universo teatral y literario de gran altura que, sorprendentemente, todavía no ha sido representado en Italia, y del que tan sólo se ha publicado un texto en una antología dedicada al teatro contemporáneo (Coco, 1998). Pero habría que enumerar una larga lista de dramaturgos reconocidos (para no extenderme, remito al lector a los manuales de teatro contemporáneo) que no aparecen en la documentación que he conseguido recopilar. Sí aparecen nombres importantes como el de Sanchis Sinisterra (del que ya se había publicado alguna obra, y existe el proyecto de que aparezcan otras), o Vallejo, y de otros más jóvenes como Tomeo, Belbel o García; estos dos últimos cuentan con algunas piezas traducidas, y como hemos visto entran en Italia a través del Teatro della Limonaia y de su festival internacional dedicado a Madrid.

También llama la atención la continuada presencia de La Cuadra y sus espectáculos supuestamente defensores de lo andaluz (tal vez habría que matizar que del ingrediente gitano). En efecto, el andalucismo parece ser una de las notas más presentes en los escenarios italianos, y en esos casos no suele faltar la alusión a García Lorca. Pero las reseñas, como hemos podido ver, mencionan también a cineastas como Buñuel y, más recientemente, a Almodóvar e incluso a Bigas Luna. Una visión muy concreta y reducida, entonces, de lo español, que sigue moviéndose en la línea de lo diverso cuando no de lo excéntrico. Cabe preguntarse hasta cuándo.

\section{REFERENCIAS BIBLIOGRÁFICAS}

BELBEL, Sergi (1996a). Dopo la pioggia. Intercity Plays 2: 162-176.

— (1996b). Morire. Intercity Plays 2: 177-196.

Coco, Emilio (1998). Teatro spagnolo contemporaneo. Alessandria: Edizioni dell'Orso. 
GARCíA, Rodrigo (2003). Sei pezzi di teatro in tanti round. Milán: Ubulibri.

GARCía RodríGuez, Coral (2003). Teatro español en Italia: Valle-Inclán, García Lorca, Buero Vallejo, Sastre y Arrabal. Florencia: Alinea.

RiPELlino, Angelo Maria (1989). Siate buffi. Cronache di teatro, circo e altre arti (“L’Espresso”, 1969-77). Roma: Bulzoni.

VillanueVA, Darío, et alii, eds. (1992). Los nuevos nombres: 1975-1990. Barcelona: Crítica; vol. 9 de la Historia y crítica de la literatura española.

Periódicos:

Avanti!, Roma.

Il Corriere della Sera, Milán.

La Gazzetta di Firenze, Florencia.

Il Giornale d’Italia, Roma. Il Giorno, Milán.

La Nazione, Florencia.

La Notte, Milán.

Provincia Pavese, Pavía.

La Repubblica, Roma. Il Tempo, Roma.

L'Umanità, Roma.

L'Unità, Roma.

La Voce, Milán.

Revistas y semanarios:

Il Patalogo.

La Repubblica, Supplemento Il Venerdì.

Sicario. 